\title{
The effects of fibroblasts on wave dynamics in a mathematical model for human ventricular tissue
}

\author{
Alok Ranjan Nayak ${ }^{1 *}$ and Rahul Pandit ${ }^{2 \dagger}$ \\ ${ }^{1}$ Robert Bosch Centre for Cyber Physical Systems, \\ Indian Institute of Science, Bangalore 560012, India. \\ ${ }^{2}$ Centre for Condensed Matter Theory, Department of Physics, \\ Indian Institute of Science, Bangalore 560012, India.
}

\begin{abstract}
We present systematic numerical studies of electrical-wave propagation in two-dimensional (2D) and three-dimensional (3D) mathematical models, for human, ventricular tissue with myocyte cells that are attached (a) regularly and (b) randomly to distributed fibroblasts. In both these cases we show that there is a parameter regime in which single rotating spiral- and scroll-wave states (RS) retain their integrity and do not evolve to a state ST that displays spatiotemporal chaos and turbulence. However, in another range of parameters, we observe a transition from ST to RS states in both 2D or 3D domains and for both cases (a) and (b). Our studies show that the ST-RS transition and rotation period of a spiral or scroll wave in the RS state depends on (i) the coupling strength between myocytes and fibroblasts and (ii) the number of fibroblasts attached to myocytes. We conclude that myocyte-fibroblast coupling strength and the number of fibroblasts are more important for the ST-RS transition than the precise way in which fibroblasts are distributed over myocyte tissue.
\end{abstract}

PACS numbers: 87.19.Hh, 89.75.Kd, 05.45.-a

Keywords: Mathematical Model, Fibroblasts, Action-potential-duration-restitution, Spiral-wave dynamics

\section{INTRODUCTION}

Approximately $16 \%$ of all deaths in the industrialized world are caused by cardiac arrhythmias like ventricular tachycardia (VT) and ventricular fibrillation (VF) $[1,2]$. There is a broad consensus that the analogs of VT and VF in mathematical models for cardiac tissue are, respectively, (a) a single rotating spiral or scroll wave of electrical activation and (b) spiral-wave or scroll-wave turbulence, which displays broken electrical waves and spatiotemporal chaos [3-6]. Thus, it is very important of study such spiral and scroll waves to develop an understanding of life-threatening cardiac arrhythmias. Such studies are truly interdisciplinary because they require inputs from biology, bio-medical engineering, cardiology, on the one hand, and physics, nonlinear dynamics, and numerical methods, on the other. We use methods from these areas to solve the nonlinear, partial-differential equations for state-of-the-art mathematical models for cardiac tissue with myocytes and fibroblasts. We build on our earlier studies of this problem $[7,8]$ to elucidate the role of two different forms of myocyte-fibroblast couplings on spiral- and scroll-wave dynamics in such models by using theoretical ideas from spatiotemporal chaos.

It is useful to begin with an overview of experimental and computational studies of cardiac myocytes and fibroblasts. Cardiac fibroblasts, which are inexcitable cells, often multiply and connect with cardiac myocytes during fibrosis [9-11], a process of cardiac-tissue healing after a myocardial infarction. Fibroblasts in cell culture and in intact tissue can couple with myocytes by expressing either the connexins-43 (Cx43) or $\mathrm{Cx} 45[12$

\footnotetext{
* alok@cps.iisc.ernet.in

† rahul@physics.iisc.ernet.in
}

16]. Zlochiver, et al. [15] have shown the expression of $\mathrm{Cx} 43$ between fibroblasts and myocytes in a monolayer of myocytes and fibroblasts of neonatal rats; Miragoli, et al. [16] have reported that $\mathrm{Cx} 43$ and $\mathrm{Cx} 45$ are expressed among fibroblasts and between fibroblasts and myocytes in cultured fibroblasts coated over rat-ventricular-myocyte strands. Both experimental and computational studies have shown that such coupling between myocytes and fibroblasts enhances electrical-signal propagation in cardiac tissue [7, 15-18]; this enhancement increases with $N_{f}$, the number of fibroblasts that are attached to a myocyte. In cell-culture experiments, Miragoli, et al. [16] have found that the conduction velocity (CV) decreases with an increase in the density of fibroblasts in cultured strands of neonatal-rat myocytes coated by fibroblasts; studies by McSpadden, et al. [17] have found that CV decreases as the fibroblast number increases on the top of a myocyte layer in a monolayer of neonatal-rat cardiac myocytes, which are electrotonically loaded with a layer of cardiac fibroblasts. Zlochiver, et al. [15] have shown that CV decreases as (i) the gapjunctional conductance increases or (ii) the fibroblasts density increases in their experiments with fibroblasts of neonatal rats; they have also obtained similar result in their computational studies in a two-dimensional (2D) sheet of myocyte tissue in the dynamic Luo-Rudy (LRd) model $[19,20]$ by inserting fibroblasts. Computational studies by Xie, et al., [18] have shown that CV decreases as they increase the gap-junctional coupling or the fibroblasts density in a 2D LR1 myocyte model [21], with either randomly attached or randomly inserted fibroblasts. In simulations with the 2D TNNP04 model (due to ten Tusscher, et al. [22]), Nayak, et al. [7] have found that CV either decreases or increases, with attached fibroblasts, as they increase the gap-junctional coupling. The experimental and computational investigations men- 
tioned above show that both the gap-junctional coupling and $N_{f}$ enhance CV and, therefore, they can play a crucial role in spiral- and scroll-wave dynamics in mathematical models for cardiac tissue.

We develop and investigate two models with different arrangements of fibroblasts that are attached to myocytes. In the first arrangement there is a regular, spatially periodic attachment of fibroblast, whereas, in the second arrangement, fibroblasts are attached randomly to myocytes. Our study has been designed to understand the effects of fibroblast organization, fibroblast density, and the myocyte-fibroblast coupling on spiral- and scrollwave dynamics. We use two parameter sets for myocytes. The first set leads to a stable rotating spiral or scroll (RS) wave; the second leads to spiral- or scroll-waveturbulence (ST) states in an isolated myocyte domain. By investigating an ST state in the presence of fibroblasts, we observe that both models, with regularly and randomly attached fibroblasts, show transitions from an ST to an RS state, depending on the myocyte-fibroblast coupling $G_{j}$ and the maximum number $N_{f}$ of fibroblasts attached to a myocyte in our simulation domain. We find that, once ST is converted to RS, the spiral or scroll rotation period increases as we increase $G_{j}$ and $N_{f}$. Our study with an RS state and fibroblasts shows that an RS remains unchanged in both models with regularly and randomly attached fibroblast; and the rotation period increases as we increase $G_{j}$ and $N_{f}$.

The remainder of this paper is organized as follows. Section II is devoted to a description of our model and the numerical methods we use. Section III is devoted to our results. Section IV contains a discussion of the significance of our results.

\section{MODEL AND METHODS}

In this Section, we describe the details of our myocytefibroblast models for two-dimensional (2D) and threedimensional (3D) tissue. We also explain the numericalsimulation techniques that we use to solve the partial differential equations (PDEs) that comprise our mathematical models. We also discuss the methods that we use to analyze the data from our numerical simulations.

\section{A. Model}

The 2D and 3D myocyte domains, with attached fibroblasts, can be modeled by the following PDEs and ordinary-differential-equations (ODEs) [23, 24]:

$$
\begin{aligned}
\frac{\partial V_{m}}{\partial t} & =\frac{-I_{m}+N_{f}(\mathbf{x}) I_{j}}{C_{m}}+D \nabla^{2} V_{m}, \\
\frac{\partial V_{f}}{\partial t} & =\frac{-I_{f}-I_{j}}{C_{f}}
\end{aligned}
$$

where

$$
I_{j}=G_{j}\left(V_{f}-V_{m}\right)
$$

here $C_{m}$ is the total cellular capacitance of a myocyte, $V_{m}$ is the myocyte transmembrane potential, i.e., the voltage difference between intra- and extra-cellular spaces, and $I_{m}$ is the sum of all the ionic currents that cross the myocyte cell membrane; $C_{f}, V_{f}$, and $I_{f}$ are, respectively, the total cellular capacitance, the transmembrane potential, and the sum of all ionic currents for the fibroblast; $N_{f}(\mathbf{x})$ is the number of identical fibroblasts attached to a myocyte in our simulation domain at the point $\mathbf{x}$; and $I_{j}, G_{j}$, and $D$ are, respectively, the gap-junctional current, the myocyte-fibroblasts gap-junctional conductance, and the diffusion coefficient that is related to the gap-junctional conductance between myocytes.

For myocytes, we use the state-of-the-art mathematical model for human ventricular tissue developed by ten Tusscher and Panfilov (the TP06 model) [25]. In the TP06 model the total ionic current is

$$
\begin{aligned}
I_{m}= & I_{N a}+I_{C a L}+I_{t o}+I_{K s}+I_{K r}+I_{K 1} \\
& +I_{N a C a}+I_{N a K}+I_{p C a}+I_{p K}+I_{b N a}+I_{b C a},
\end{aligned}
$$

where $I_{N a}$ is the fast, inward $\mathrm{Na}^{+}$current, $I_{\mathrm{CaL}}$ the Ltype, slow, inward $\mathrm{Ca}^{2+}$ current, $I_{t o}$ the transient, outward current, $I_{K s}$ the slow, delayed, rectifier current, $I_{K r}$ the rapid, delayed, rectifier current, $I_{K 1}$ the inward, rectifier $\mathrm{K}^{+}$current, $\mathrm{I}_{\mathrm{NaCa}}$ the $\mathrm{Na}^{+} / \mathrm{Ca}^{2+}$ exchanger current, $I_{\mathrm{NaK}}$ the $\mathrm{Na}^{+} / \mathrm{K}^{+}$pump current, $I_{p \mathrm{Ca}}$ and $I_{p K}$ the plateau $\mathrm{Ca}^{2+}$ and $\mathrm{K}^{+}$currents, and $I_{b N a}$ and $I_{b C a}$ the background $\mathrm{Na}^{+}$and $\mathrm{Ca}^{2+}$ currents, respectively. The full sets of equations for this model, including the ODEs for the ion-channel gating variables and the ion dynamics, are given in Refs. [7, 26].

We follow MacCannell, et al. [27] to model the fibroblasts as passive elements. The fibroblast ionic current $I_{f}$ is

$$
I_{f}=G_{f}\left(V_{f}-E_{f}\right),
$$

where $G_{f}$ and $E_{f}$ are, respectively, the conductance and the resting membrane potential for the fibroblast.

Physical units in our model are as follows: time $t$ is in milliseconds (ms), $V_{m}$ and $V_{f}$ are in millivolts $(\mathrm{mV})$, $C_{m}$ and $C_{f}$ are in picofarads $(\mathrm{pF}), I_{m}$ and $I_{f}$ are in picoamperes (pA), $G_{f}$ is in nanoSiemens $(\mathrm{nS}), E_{f}$ is in $\mathrm{mV}, G_{j}$ is in $\mathrm{nS}$, and $D$ is in $\mathrm{cm}^{2} / \mathrm{ms}$.

We study models with (a) regularly attached fibroblasts and (b) randomly attached fibroblasts. In case (a), $N_{f}(\mathbf{x})=N_{f}$ for all site $\mathbf{x}$ in our simulation domain. In case (b) we choose $N_{f}(\mathbf{x})$ randomly at each site $\mathbf{x} ; N_{f}(\mathbf{x})$ can be any integer from 0 to $N_{f}$, with equal probability for any one of these values.

\section{B. Methods}

Our 2D and 3D simulation domains are, respectively, squares $(1024 \times 1024$ grid points $)$ and rectangular parallelepipeds $(1024 \times 1024 \times 8$ grid points $)$. We use 5 -point and 7-point stencils for the Laplacian in 2D and 3D, respectively, and a finite-difference scheme with step sizes $\delta x=\delta y=0.25 \mathrm{~mm}$ in $2 \mathrm{D}$, and $\delta x=\delta y=\delta z=0.25 \mathrm{~mm}$ in 
$3 \mathrm{D}$, i.e., our simulation domains are $256 \times 256 \mathrm{~mm}^{3}$ (in $2 \mathrm{D})$ and $256 \times 256 \times 2 \mathrm{~mm}^{2}$ (in $3 \mathrm{D}$ ). For time marching we use a forward-Euler scheme with $\delta t=0.02 \mathrm{~ms}$. We use Neumann (no-flux) conditions at the boundaries of our simulation domain.

For numerical efficiency, we have carried out our simulations on parallel computers, with an MPI code that we have developed for the TP06 model. Our code divides the 2D (or 3D) simulation domain into $n$ columns (or slabs) along the $x$-direction of the domain, i.e., each processor carries out the computations for $(1024 / n) \times 1024$ and $(1024 / n) \times 1024 \times 16$ grid points, respectively, for $2 \mathrm{D}$ and $3 \mathrm{D}$ domains. To compute the Laplacian at the interface of processor boundaries, we use two extra grid lines (or surfaces), which can send and receive the data from left- and right-neighbor processors. The Neumann boundary condition is taken care of by adding an extra layer of grid points on the boundaries of the simulation domain of each processor.

Reference [28] suggests that we must have $D \delta t /\left(\delta x^{2}\right)<$ $1 / 2 d$ for numerical stability, where $d$ is the dimension of the simulation domain. For the TP06 model, with diffusion coefficients $D=0.00154 \mathrm{~cm}^{2} / \mathrm{ms}$ [25], time step $\delta t=0.02 \mathrm{~ms}$, and space step $\delta x=0.25 \mathrm{~mm}$, the value of $D \delta t /(\delta x)^{2}$ is $\simeq 0.05$; for the TP06 model, the quantity $1 / 2 d=0.25$ and $\simeq 0.17$, for the $2 \mathrm{D}$ and $3 \mathrm{D}$ domains, respectively, i.e., we have numerical stability because $D \delta t /(\delta x)^{2}<1 / 2 d$.

We check the accuracy of our numerical scheme, as in Ref. [28], by varying both $\delta t$ and $\delta x$ in a cable-type domain of myocytes $[7,26]$ and by measuring $\mathrm{CV}$ of a plane wave, which is injected into the domain by stimulating its left boundary for $3 \mathrm{~ms}$ with a stimulus of strength $150 \mathrm{pA}$. With $\delta x=0.25 \mathrm{~mm}, \mathrm{CV}$ increases by $1.1 \%$ when we change $\delta t$ from 0.02 to $0.01 \mathrm{~ms}$; if we decrease $\delta x$ from 0.25 to $0.15 \mathrm{~mm}$, with $\delta t=0.02 \mathrm{~ms}$, then CV increases by $3.1 \%$; these changes are similar to those found in other studies [22, 26, 28, 29].

Although the numerical method we use satisfies both numerical-stability and accuracy conditions, an inappropriately large $\delta x$ can give irregular wavefront-curvature, as a consequence of numerical artifacts [7, 26, 28]; this leads to unphysical wave dynamics. We check that our results are free from such numerical artifacts by investigating the spatiotemporal evolution of an expanding wave front that emerges from a point stimulus. We find that fronts of the expanding wave do not deviate substantially from circles, when we apply a point stimulus of strength $450 \mathrm{pA}$ for $3 \mathrm{~ms}$ at the center of the domain.

We use two parameter sets P1 and P2 for myocytes to obtain, respectively, a stable rotating spiral (RS) and a spiral-turbulence (ST) states in our 2D simulation domain, and a stable rotating scroll or scroll-wave turbulence in our $3 \mathrm{D}$ domain. The parameter set $\mathrm{P} 1$ is the original one used in the TP06 model $[7,25,26]$. In the P2 parameter set, we use the following parameters, with all other parameters the same as in the original TP06 model: (a) $G_{K r}$, the $I_{K r}$ conductance, is $0.172 \mathrm{nS} / \mathrm{pF}$; (b) $G_{K s}$, the $I_{K s}$ conductance, is $0.441 \mathrm{nS} / \mathrm{pF}$; (c) $G_{p C a}$, the $I_{p C a}$ conductance, is $0.8666 \mathrm{nS} / \mathrm{pF}$; (d) $G_{p K}$, the $I_{p K}$ conductance, is $0.00219 \mathrm{nS} / \mathrm{pF}$; and (e) $\tau_{f}$, the time constant of the $f$ gating variable that is associated with the $I_{C a L}$ current, is increased 2 times compared to its value in the TP06 model $[7,25,26]$. Our fibroblasts parameters are as follows: $C_{f}=6.3 \mathrm{pF}, G_{f}=4 \mathrm{nS}, E_{f}=-49.0 \mathrm{mV}$, and $G_{j}$ in the range $0 \leq G_{j} \leq 6 \mathrm{nS}[7,30]$.

To obtain spiral and scroll waves we use the S1-S2 cross-field protocol $[26,31,32]$. We apply a stimulus (S1) of strength $150 \mathrm{pA}$ for $3 \mathrm{~ms}$ to the left boundaries of our simulation domains, to form a plane wave. We then apply the second (S2) stimulus, with the same strength and duration as the $\mathrm{S} 1$ stimulus, from the bottom boundary and with $0 \mathrm{~mm} \leq y \leq 125 \mathrm{~mm}$ in $2 \mathrm{D}$, and $0 \mathrm{~mm} \leq y \leq 125 \mathrm{~mm}$ and $0 \mathrm{~mm} \leq z \leq 2 \mathrm{~mm}$ in $3 \mathrm{D}$. This protocol leads to the formation of spiral and scroll waves, respectively, in our $2 \mathrm{D}$ and $3 \mathrm{D}$ domains.

To examine the spatiotemporal evolution of our system, we obtain pseudocolor or isosurface plots of $V_{m}$, time series of $V_{m}$, from representative points $(x=125$ $\mathrm{mm}, y=125 \mathrm{~mm}$ for $2 \mathrm{D}$, and $x=125 \mathrm{~mm}, y=125$ $\mathrm{mm}, z=1.25 \mathrm{~mm}$ for $3 \mathrm{D}$ ), which we mark with an asterisk $(*)$ in all pseudocolor plots of $V_{m}$. We examine the inter-beat interval (IBI), by using this time series with $4.4 \times 10^{5}$ data points; the IBI is the interval between two successive spikes in this time series. We obtain the power spectra $E(\omega)$, of the time series of $V_{m}$, by using $2 \times 10^{5}$ data points; to eliminate transients we remove the initial $2.4 \times 10^{5}$ data points. To obtain the rotation period $\mathrm{T}$ of a spiral, in an RS state, we average over the last 5 rotations of that RS.

\section{RESULTS}

In subsection III A, we begin by studying spiral-wave dynamics in a 2D domain of myocytes without fibroblasts; we then introduce fibroblasts, either regularly or randomly, and examine the effects they have on spiralwave dynamics. Subsection III B contains the results of our studies of scroll-wave dynamics in our 3D simulation domain.

\section{A. Spiral-wave dynamics in our $2 \mathrm{D}$ model}

In Fig. 1(A), we show a pseudocolor plot of $V_{m}$, at time $t=8.8 \mathrm{~s}$, for the parameter set $\mathrm{P} 1$, in our $2 \mathrm{D}$ simulation domain without fibroblasts; the initial condition evolves to a state with a single rotating spiral (RS). The local time series of $V_{m}(x, y, t)$, from the representative point shown by the asterisk in Fig. 1(A)), is given in Fig. 1(B) for $0 \mathrm{~s} \leq t \leq 8.8 \mathrm{~s}$; a plot of the IBI versus the beat number is given in Fig. 1(C), which shows that, after initial transients, the spiral wave rotates periodically with an average rotation period $T \simeq 212 \mathrm{~ms}$. The power spectrum $E(\omega)$ in Fig. 1(D) has discrete peaks at the fundamental frequency $\omega_{f} \simeq 4.75 \mathrm{~Hz}$ and its harmonics. The periodic time series of $V_{m}$, the flattening of the IBI, and the discrete peaks in $E(\omega)$ demonstrate that the time evolution of the spiral wave, with the P1 parame- 

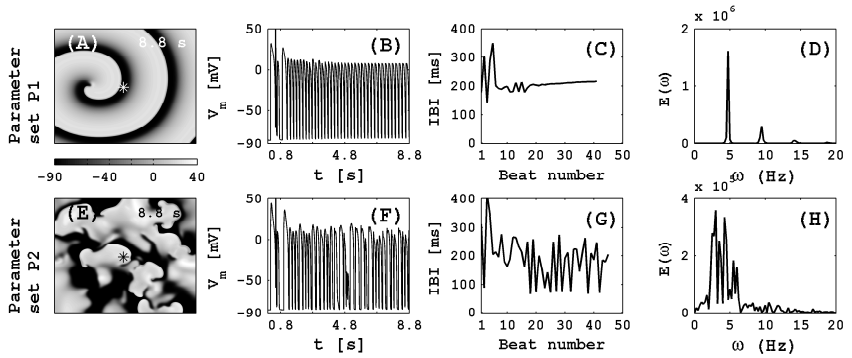

FIG. 1. The rotating-spiral (RS) and spiral-turbulence (ST) states in a $2 \mathrm{D}$ domain in the absence of fibroblasts. For the parameter set $\mathrm{P} 1$, the pseudocolor plot of $V_{m}$ in (A), the periodic nature of the local time series for $V_{m}$ from the representative point (marked by an asterisk $*$ in $(\mathrm{A})$ ) in (B), the flattening IBI with an average rotation period $T \simeq 212 \mathrm{~ms}$ in (C), and the discrete peaks in the power spectrum with the fundamental frequency $\omega_{f}=4.75 \mathrm{~Hz}$ and its harmonics in (D) characterize the RS state. The exact analogs of plots (A)(D) are shown, respectively, in (E)-(H) for the $\mathrm{P} 2$ parameter set; the irregular local time series, the fluctuating behavior of the IBI and the broad-band nature of the power spectrum characterize the ST state.

ter set, is periodic. In Figs. 1(E)-(H), we show the exact analogs of Figs. 1(A)-(B) for the P2 parameter set. The non-periodic local time series in Fig. 1(F), the fluctuating IBI in Fig. $1(\mathrm{G})$, and the broad-band nature of $E(\omega)$ in Fig. 1(H) are characteristic of the ST state. The pseudocolor plot in Fig. 1(E), at time $t=8.8 \mathrm{~s}$, shows such an ST state, which arises from the steep slope of the actionpotential-duration-restitution (APDR) plot [8, 25]. In summary, then, in the absence of fibroblasts, the parameter sets $\mathrm{P} 1$ and $\mathrm{P} 2$ lead, respectively, to (a) an RS state and (b) an ST state in our 2D simulation domain.

We now examine the effects of fibroblasts on spiralwave dynamics in both RS and ST states. We begin our investigation with the $\mathrm{P} 1$ parameter set and with the regularly attached fibroblast model with $1 \leq N_{f} \leq 6$ and $1 \mathrm{nS} \leq G_{j} \leq 8 \mathrm{nS}$; the remaining parameters for fibroblasts are as in subsection II B.

In Figs. 2(A1)-(A4) we show pseudocolor plots of $V_{m}$, at time $t=8.8 \mathrm{~s}$, with the $\mathrm{P} 1$ parameter set for regularly attached fibroblast model with $G_{j}=4 \mathrm{nS}$ and different values of $N_{f}$. The analogs of Figs. 2(A1)-(A4) are shown in Figs. 2(B1)-(B4) for the P2 parameter set. The plots in the first row of Fig. 2 show that the RS state, which we obtain in the absence of fibroblasts, does not evolve into an ST state; however, the spiral-arm width $W_{d}$ decreases as we increase $N_{f}$, for a fixed value of $G_{j}$ (first row of Fig. 2). We define $W_{d}$ to be the difference of the radial distance between the wave front and the wave back of a spiral arm, whose center is located at the spiral core. Such a decrease of $W_{d}$ is related to the shortening of the action-potential-duration (APD) of a myocyte-fibroblast composite that has been discussed in Refs. [7, 8].

For the P2 parameter set, we observe a transition from an ST to an RS state as we increase $N_{f}$ for a fixed value of $G_{j}$ (second row of Fig. 2). Such an ST-RS transition is the consequence of the suppression of the steep APDR

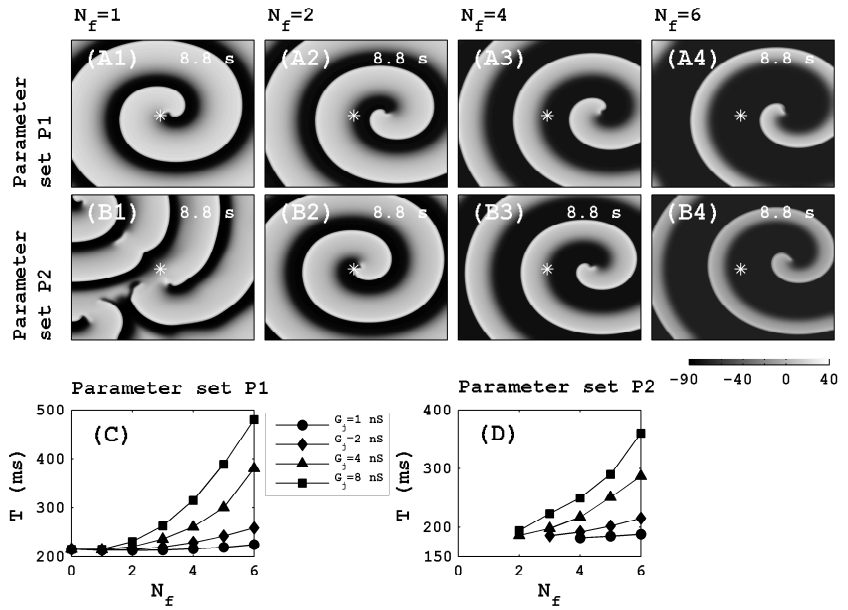

FIG. 2. Various RS and ST states in our 2D domain for the regularly attached fibroblast model. ((A1)-(B4)) Pseudocolor plots of $V_{m}$, with $G_{j}=4 \mathrm{nS}$ for $N_{f}=1,2,4$, and 6 , illustrate that the RS state (parameter set P1) remains in an RS state as $N_{f}$ increases (first row). However, an ST state (parameter set P2) shows a transition to an RS state as $N_{f}$ increases (second row). ((C)-(D)) The rotation period T of RS increases as $N_{f}$ increases, for a fixed value of $G_{j}$, and vice-versa, for both the $\mathrm{P} 1$ and $\mathrm{P} 2$ parameter sets.

slope of a myocyte-fibroblast composite at the cellular level $[8,33]$. Once the ST state is suppressed, a single spiral in an RS state rotates periodically as shown Figs. 2((B2)-(B4)). In Figs. 2(C) and (D), we plot, respectively, the rotation period $\mathrm{T}$ of a spiral wave in an RS state versus $N_{f}$, for different values of $G_{j}$, for the $\mathrm{P} 1$ and $\mathrm{P} 2$ parameter sets. We find that $\mathrm{T}$ increases as (i) $N_{f}$ increases, with a fixed value of $G_{j}$, and (ii) $G_{j}$ increases, with a fixed value of $N_{f}$. This increase of T is a consequence of the decrease of $\mathrm{CV}$ that is associated with a decrease of the upstroke velocity of a myocytefibroblast composite AP in its depolarization phase, as shown in Refs. [7, 8, 18]. Furthermore, we observe that the minimum value of $N_{f}$, required for the ST-RS transition, decreases as $G_{j}$ increases, for the P2 parameter set.

We focus next on spiral-wave dynamics, with P1 and P2 parameter sets, in our randomly attached fibroblast model. In Fig. 3, we show the exact analogs of Fig. 2, but now for the randomly attached fibroblast model. The pseudocolor plots in Figs. 3(A1)-(A4) show that the randomness in attaching fibroblasts does not lead to an RSST transition for the P1 parameter set. However, inspite of the randomness in the arrangement of fibroblasts, we observe an ST-RS transition for the P2 parameter set (see Figs. 3(B1)-(B4)), which is qualitatively similar to the ST-RS transition in the P1 case (compare the second rows of Figs. 2 and 3 ). However, the minimum value of $N_{f}$, required for an ST-RS transition, is higher for the randomly attached fibroblast model than in the regularly attached case (compare Figs. 2(D) and 3(D)). 

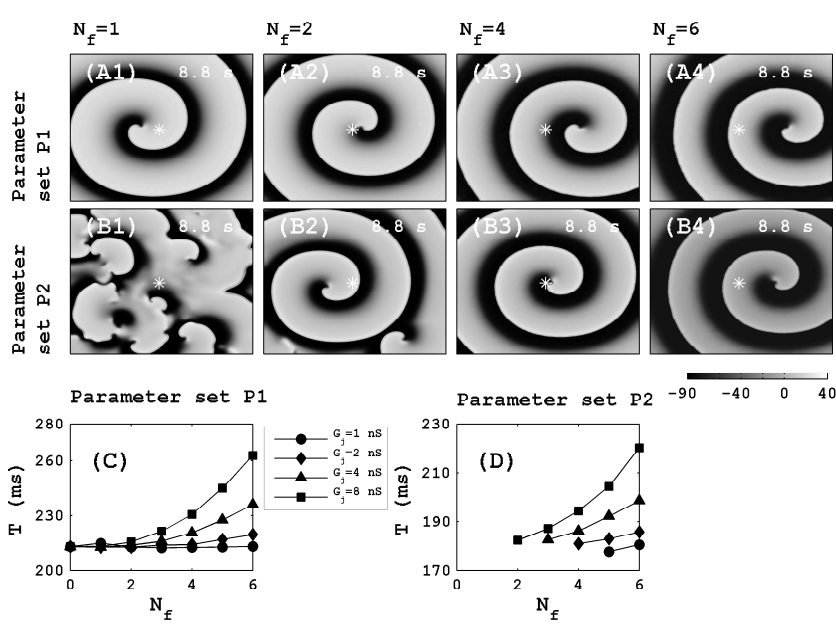

FIG. 3. Various RS and ST states in our 2D domain for the randomly attached fibroblast model (this figure is the analog of Fig. 2). The results are qualitatively similar to those in Fig. 2 for the regularly attached fibroblast case. Note that the minimum value of $N_{f}$, for a fixed value of $G_{j}$, for the ST-RS transition, is higher compared to that in Fig. 2(D).

\section{B. Scroll-wave dynamics in our 3D model}

We turn now to a systematic study of scroll-wave dynamics in our $3 \mathrm{D}$ simulation domain. For both the P1 and P2 parameter sets and both regularly and randomly attached fibroblast models, we carry out simulations to study the dependence of scroll-wave dynamics on $N_{f}$ and $G_{j}$. We present our numerical results below.

In Figs. 4(A1), (A2), (A3) and (A4), we show, respectively, isosurface plots of $V_{m}$, at time $t=8.8 \mathrm{~ms}$, for the P1 parameter set in our regularly attached fibroblast model with $G_{j}=4 \mathrm{nS}$ and $N_{f}=0$ (i.e., isolated myocytes), $N_{f}=1, N_{f}=2$, and $N_{f}=4$. In the absence of fibroblasts, i.e., $N_{f}=0$, the $\mathrm{P} 1$ parameter set displays a rotating scroll wave with fundamental frequency $\omega_{f} \simeq 5 \mathrm{~Hz}$ and rotation period $\mathrm{T} \simeq 201 \mathrm{~ms}$; this is consistent, because $\omega_{f} \simeq 1 / \mathrm{T}$. In Fig. $4(\mathrm{C})$, we plot T versus $N_{f}$ for $G_{j}=1 \mathrm{nS}(\bullet)$ and $4 \mathrm{nS}(\bullet)$. We find that T increases as we increase (i) $N_{f}$, for a fixed value of $G_{j}$, or (ii) $G_{j}$, for a fixed value of $N_{f}$. In Figs. $4((\mathrm{~B} 1)-(\mathrm{B} 4))$ and (D), we show, respectively, the exact analogs of Figs. 4((A1)(A4)) and (C), for the P2 parameter set. In the absence of fibroblasts and for the P2 parameter set, we obtain a scroll-wave-turbulence state (Fig. 4(B1)); this scrollwave turbulence is converted to a rotating scroll if we have $N_{f}>1$ (second row of Fig. 4). Once the scroll-wave turbulence state is suppressed, a rotating scoll rotates with a period $\mathrm{T}$, which increases as we increase $N_{f}$ for a fixed value of $G_{j}$, and vice-versa (Fig. 4(D)). Furthermore, from Fig. 4(D), we find that the minimum value of $N_{f}$, required for the ST-RS transition, is 4 and 2, respectively, for $G_{j}=1 \mathrm{nS}(\bullet)$ and $4 \mathrm{nS}(\bullet)$. The isosurface plots in Fig. 4 show that the width $W_{d}$ of a scroll-wave arm in the rotating-scroll state decreases as we increase $N_{f}$ for both the $\mathrm{P} 1$ and $\mathrm{P} 2$ parameter sets. The mecha-
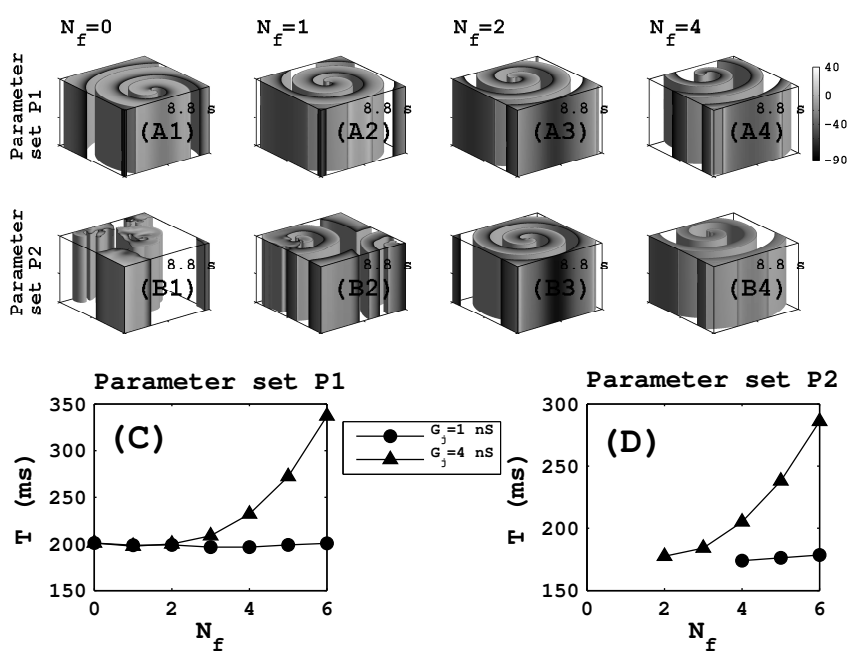

FIG. 4. The rotating-scroll and scroll-wave-turbulence states, in our 3D simulation domain of size $256 \times 256 \times$ $2 \mathrm{~mm}^{3}$, for the regularly attached fibroblast model, are shown in ((A1)-(B4)) via isosurface plots of $V_{m}$. The myocytefibroblast coupling strength $G_{j}=4 \mathrm{nS}$. The scroll-arm width of a rotating scroll, with the $\mathrm{P} 1$ parameter set, decreases as $N_{f}$ increases (first row). The scroll-wave turbulence, associated with the P2 parameter set, is converted to a rotating scroll as $N_{f}$ increases (second row). ((C)-(D)) Plots of the rotation period $\mathrm{T}$ of a scroll wave in a rotating-scroll state, for the P1 and P2 parameter sets; for both parameter sets $\mathrm{T}$ increases as $G_{j}$ increases; note that, for the ST-RS transition, the minimum value of $N_{f}$ decreases as $G_{j}$ increases.
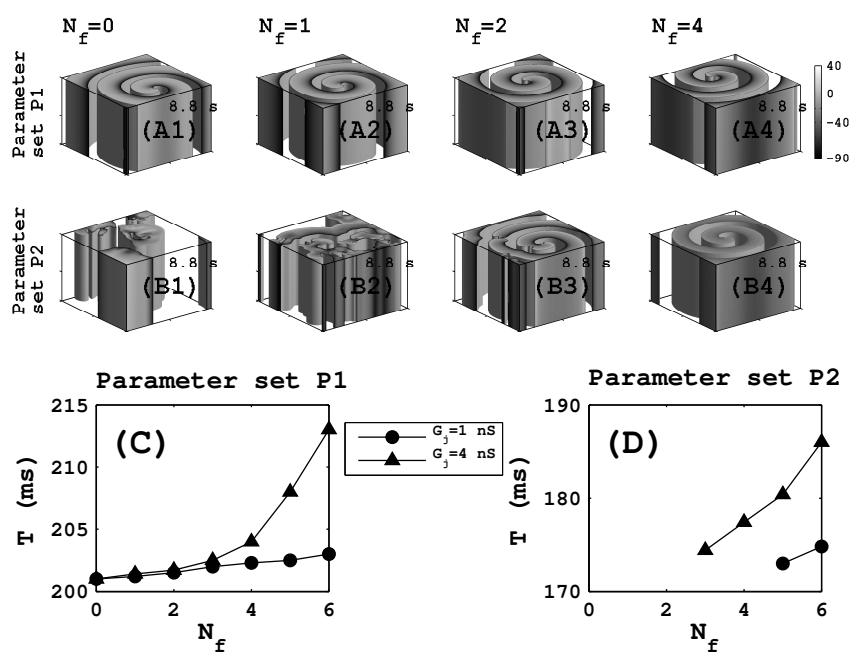

FIG. 5. The rotating-scroll and scroll-wave-turbulence states, in our 3D simulation domain for the randomly attached fibroblast model; the exact analog of Fig. 4. The results are qualitatively similar to those for the regularly attached fibroblast case. Note that the ST-RS transition $N_{f}$ value, for a fixed value of $G_{j}$, is higher than its counterpart in Fig. 4(D). 
nisms of the ST-RS transition, and increase of T and a decrease of $W_{d}$, as we increase $N_{f}$ and $G_{j}$, are the same as those we have found in our 2D studies.

In Fig. 5 we show the exact analog of Fig. 4 for the randomly attached fibroblast model, with both the P1 and P2 parameter sets. Our scroll-wave results here are similar to those for the case of regularly attached fibroblast model. From Fig. 5(D), we find that the minimum value of $N_{f}$, for the ST-RS transition, is 5 and 3, respectively, for $G_{j}=1 \mathrm{nS}(\bullet)$ and $4 \mathrm{nS}(\boldsymbol{\bullet})$. Note that this minimum value of $N_{f}$ is higher for the randomly attached fibroblast model than it is for the regularly attached fibroblast model (compare Figs.4(D) and 5(D)).

\section{CONCLUSIONS}

We have presented the most extensive numerical study carried out so far of the effects of fibroblasts on spiraland scroll-wave dynamics in a mathematical model for human ventricular tissue with fibroblasts, attached regularly or randomly to myocytes. Our numerical study has been designed to uncover the role of (i) the organization of fibroblasts in ventricular tissue (i.e., to compare regular and random arrangements), (ii) myocyte-fibroblast coupling $G_{j}$, and (iii) the density of fibroblasts, i.e., the maximum number of fibroblasts $N_{f}$ attached to a myocyte. One of the principal results of our studies is that spiral- and scroll-wave dynamics depend only slightly on the details of the organization of fibroblasts in ventricular tissue. However, the ST-RS transition, the stability of spiral- and scroll-wave turbulence, the rotation period of a rotating spiral and scroll, and the width of a rotating spiral and scroll arms, depend sensitively on $N_{f}$ and $G_{j}$.

Earlier studies have investigated the effects of fibroblasts on spiral-wave dynamics by introducing randomly diffuse fibroblasts in a myocyte domain [15, 34]. Such randomly diffuse fibroblasts in a myocyte domain inhibit electrical-wave propagation, and initiate spiral-wave turbulence state. Studies by Xie, et al [18] have found that spiral-wave breakup occurs, in an LR1 model, because of randomly diffuse fibroblasts in a localized area of a simulation domain; Zlochiver, et al. [15] have shown from their experiments and simulations that a rotating spiral becomes unstable and, finally, spiral breakup occurs, as they increase the percentage of diffuse fibroblasts. Majumder, et al. [34] have shown from their numerical experiments that a transition from an RS to various ST states occurs depending on the percentage of fibroblasts in their simulation domain. In our attached-fibroblast model studies, fibroblasts do not inhibit wave propagation [7]; however, fibroblasts attached to a myocyte can lower the steepness of the APDR curve, depending on the values of $G_{j}$ and $N_{f}[8,33]$. Such a lowering of the steep slope of the APDR eliminates spiral- and scroll-wave turbulence in our 2D and 3D simulation domains [35-38]. Therefore, we observe an ST-RS transition. Earlier studies in Ref. [33] have observed ST-RS spiral-wave transitions because of a suppression of the steep portion of the APDR slope in a 3D model consisting of myocytes, fibroblasts, and extracellular space by using the LR1 model [21]. However, those studies have not investigated the spiral- and scroll-wave transition as a function of $N_{f}$ and $G_{j}$. Our study shows that both $N_{f}$ and $G_{j}$ are important factors during the fibrosis process $[10,39]$.

We suggest that our results from in silico studies can be verified in in vitro experiments. Furthermore, by using advanced cell-culture techniques [40-42], our 2D and $3 \mathrm{D}$ numerical results can be tested easily in cell-culture experiments.

\section{ACKNOWLEDGMENTS}

We thank the Department of Science and Technology (DST), India, the University Grants Commission (UGC), India, and the Robert Bosch Centre for Cyber Physical Systems (RBCCPS), IISc, for support.
[1] A. S. Go, D. Mozaffarian, V. L. Roger, E. J. Benjamin, J. D. Berry, M. J. Blaha, S. Dai, E. S. Ford, C. S. Fox, S. Franco, et al., Circulation 129, e28 (2014).

[2] D. P. Zipes and H. J. Wellens, Circulation 98, 2334 (1998).

[3] J. M. Davidenko, A. V. Pertsov, R. Salomonsz, W. Baxter, and J. Jalife (1992).

[4] A. M. Pertsov, J. M. Davidenko, R. Salomonsz, W. T. Baxter, and J. Jalife, Circulation research 72, 631 (1993).

[5] R. A. Gray, A. M. Pertsov, and J. Jalife, Nature 392, 75 (1998).

[6] J. Jalife, R. A. Gray, G. E. Morley, and J. M. Davidenko, Chaos: An Interdisciplinary Journal of Nonlinear Science 8, 79 (1998).

[7] A. R. Nayak, T. Shajahan, A. Panfilov, and R. Pandit, PLoS One 8, e72950 (2013).

[8] A. R. Nayak and R. Pandit, Physical Review E 92, 032720 (2015).
[9] G. Krenning, E. M. Zeisberg, and R. Kalluri, Journal of cellular physiology 225, 631 (2010).

[10] A. Biernacka and N. G. Frangogiannis, Aging and disease 2, 158 (2011).

[11] C. A. Souders, S. L. Bowers, and T. A. Baudino, Circulation research 105, 1164 (2009).

[12] M. Rook, A. Van Ginneken, B. e. de Jonge, A. El Aoumari, D. Gros, and H. Jongsma, American Journal of Physiology-Cell Physiology 263, C959 (1992).

[13] P. Camelliti, G. P. Devlin, K. G. Matthews, P. Kohl, and C. R. Green, Cardiovascular research 62, 415 (2004).

[14] L. Chilton, W. R. Giles, and G. L. Smith, The Journal of physiology 583, 225 (2007).

[15] S. Zlochiver, V. Munoz, K. L. Vikstrom, S. M. Taffet, O. Berenfeld, and J. Jalife, Biophysical journal 95, 4469 (2008).

[16] M. Miragoli, G. Gaudesius, and S. Rohr, Circulation research 98, 801 (2006). 
[17] L. C. McSpadden, R. D. Kirkton, and N. Bursac, American Journal of Physiology-Cell Physiology 297, C339 (2009).

[18] Y. Xie, A. Garfinkel, P. Camelliti, P. Kohl, J. N. Weiss, and Z. Qu, Heart Rhythm 6, 1641 (2009).

[19] C.-h. Luo and Y. Rudy, Circulation research 74, 1071 (1994).

[20] C.-H. Luo and Y. Rudy, Circulation Research 74, 1097 (1994).

[21] C.-h. Luo and Y. Rudy, Circulation research 68, 1501 (1991).

[22] K. Ten Tusscher, D. Noble, P. Noble, and A. Panfilov, American Journal of Physiology-Heart and Circulatory Physiology 286, H1573 (2004).

[23] J. Keener and J. Sneyd, Mathematical physiology, interdisciplinary applied mathematics 8 (1998).

[24] A. V. Panfilov, A. V. Holden, et al., Computational biology of the heart (Wiley, 1997).

[25] K. Ten Tusscher and A. Panfilov, Am J Physiol Heart Circ Physiol 291, H1088 (2006).

[26] A. R. Nayak, Ph.D. thesis, Indian Institute of Science (2013).

[27] K. A. MacCannell, H. Bazzazi, L. Chilton, Y. Shibukawa, R. B. Clark, and W. R. Giles, Biophysical journal 92, 4121 (2007).

[28] R. Clayton and A. Panfilov, Progress in biophysics and molecular biology 96, 19 (2008).

[29] T. Shajahan, A. R. Nayak, and R. Pandit, PLoS One 4, e4738 (2009).

[30] Y. Xie, A. Garfinkel, J. N. Weiss, and Z. Qu, American Journal of Physiology-Heart and Circulatory Physiology 297, H775 (2009).
[31] A. R. Nayak and R. Pandit, Frontiers in physiology 5, 207 (2014).

[32] R. Majumder, A. R. Nayak, and R. Pandit, in Heart Rate and Rhythm (Springer, 2011), pp. 269-282.

[33] V. S. Petrov, G. V. Osipov, and J. Kurths, Chaos: An Interdisciplinary Journal of Nonlinear Science 20, 045103 (2010).

[34] R. Majumder, A. R. Nayak, and R. Pandit, PloS one 7, e45040 (2012).

[35] Z. Qu, J. N. Weiss, and A. Garfinkel, Physical Review E 61, 727 (2000).

[36] Z. Qu, F. Xie, A. Garfinkel, and J. N. Weiss, Annals of biomedical engineering 28, 755 (2000).

[37] J. N. Weiss, P.-S. Chen, Z. Qu, H. S. Karagueuzian, and A. Garfinkel, Circulation Research 87, 1103 (2000).

[38] A. Garfinkel, Y.-H. Kim, O. Voroshilovsky, Z. Qu, J. R. Kil, M.-H. Lee, H. S. Karagueuzian, J. N. Weiss, and P.-S. Chen, Proceedings of the National Academy of Sciences 97, 6061 (2000).

[39] T. P. Nguyen, Z. Qu, and J. N. Weiss, Journal of molecular and cellular cardiology 70, 83 (2014).

[40] Y. Haraguchi, T. Shimizu, T. Sasagawa, H. Sekine, K. Sakaguchi, T. Kikuchi, W. Sekine, S. Sekiya, M. Yamato, M. Umezu, et al., Nature protocols 7, 850 (2012).

[41] T. Shimizu, M. Yamato, Y. Isoi, T. Akutsu, T. Setomaru, K. Abe, A. Kikuchi, M. Umezu, and T. Okano, Circulation research 90, e40 (2002).

[42] T. A. Baudino, A. McFadden, C. Fix, J. Hastings, R. Price, and T. K. Borg, Microscopy and microanalysis 14, 117 (2008). 